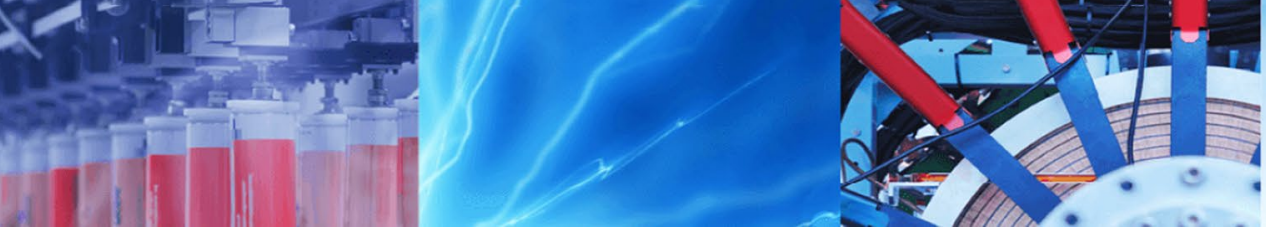

Research Article

\title{
Numerical investigation of magnetic field effect on nanofluid heat transfer in microchannel with hydrophobic surface using incompressible pre conditioned lattice Boltzmann method
}

\author{
Reza Boushehri ${ }^{1}$ Hamid Hassanzadeh Afrouzi ${ }^{2}$ (D) Mousa Farhadi ${ }^{2}$
}

Received: 10 December 2019 / Accepted: 22 May 2020 / Published online: 11 June 2020

(c) Springer Nature Switzerland AG 2020

\begin{abstract}
The flow concerning water/alumina nanofluid is studied by pre-conditioned incompressible version of Lattice Boltzmann method. The geometry at hand is a rectangular microchannel. The volume fraction of the nanoparticles is in the range of $0-2 \%$. The present study aims to combine the heat transfer enhancement methods such as application of smooth surfaces with a slip velocity coefficient of $0-0.06$ and linear magnetic field with Hartmann number of $0-40$. The results of this study show that as the wall slip increases, the amount of solid blocking the fluid motion decreases. Hence, the fluid moves with less momentum drop and it moves the path along the microchannel with less momentum dissipation. Upon applying a linear descending magnetic field to a flow, the Lorentz force and the flow behavior will change. It can also be concluded that Lorentz force reduces the slippage of the fluid layers. This will eventually reduce the effects of the hydrodynamic boundary-layer and the formation of the viscous regions. As this coefficient increases, the fluid flows easier, and the effects of the isotherms penetration in the central region of the flow can be smooth. The wall slip presence in the microchannel has also boosted the motion of the fluid and reduced the velocity gradients due to the presence of solid surfaces.
\end{abstract}

Keywords Laminar nanofluid flow · Heat transfer · Linear-decreasing magnetic field · Lattice Boltzmann method (LBM)

\section{Introduction}

In recent years, many researches have been focused on using nanofluids to modify the heat transfer rate in microchannels [1]. Nanofluids are kind of mixture fluids which contain dispersed solid particles [2]. Speaking of heat transfer enhancement, using magnetic field is another method that has been widely used [3].

Using a transverse magnetic field and nanofluid are effective measures to enhance the heat transfer in microchannels. However, these strategies result in excessive wall shear stress and unwantedly increase the pumping power required to drive the flow within microchannel. A smart solution to reduce the excessive wall shear stress is using hydrophobic wall surfaces where a tradeoff with other factors and optimization becomes important. This combination (magnetic field, nanofluid, hydrophobic wall) in some cases will result in negative consequences such as viscous dissipation and Joule heating effects.

In this regard, Aminossadati et al. [4] made a case for the study of Magneto Hydro-dynamic (MHD) nanofluid heat transfer in a microchannel. They observed that as the concentration of nanoparticles increases, the Nusselt number will rise. Concerning microfluids, Morini [5] studied the viscous dissipations of liquids and presented a criterion for this problem. He focused on the effect of the cross-section

\footnotetext{
$\triangle$ Hamid Hassanzadeh Afrouzi, h.h.afrouzi@nit.ac.ir; hamidhasanzade@yahoo.com | ${ }^{1}$ Civil and Environmental Engineering Department, Amirkabir University of Technology, Tehran, Islamic Republic of Iran. ${ }^{2}$ Faculty of Mechanical Engineering, Babol Noshirvani University of Technology, P.O. Box 484, Babol, Islamic Republic of Iran.
} 
shape and a minimum Reynolds number. Hung [6] studied the viscous dissipations effect on forced convection of nanofluid in a microtube. Azad et al. [7] examined the MHD mixed convection in a channel. Tretheway and Meinhart [8] analyzed the water flow in a microchannel. The velocity slip in the experiment was approximately 10 percent of the maximum velocity. In another study, the forced convective heat transfer of alumina-water nanofluid was analyzed by Akbarinia et al. [9]. The microchannel of the interest in their research was combined of parallel plates. They stated that the most important role in increasing Nusselt number was played by Reynolds number. Karimipour and Afrand [10] analyzed the MHD flow and heat transfer. They reported that the heat transfer rate increases with the increase of Hartmann number, Reynolds number and volume fraction of nanoparticles. In a numerical study, Karimipour et al. [11] analyzed a microchannel with nanofluid. They concluded that the Nusselt number decreases with the increase of slip coefficient. Abbaszadeh et al. [12] investigated the convective heat transfer of nanofluids in microchannel using the MHD. After a thorough study, they pointed out that the rise of Nusselt number and also entropy generation is mainly due to the increase of Reynolds number, the Hartmann number and the volume fraction of nanoparticles. Hamdan et al. [13] studied the viscous dissipations with velocity slip and temperature jump conditions on magnetic forced convective heat transfer between two parallel plates. Although there are several papers concerning with the microchannel heat transfer utilizing nanofluids such as Ref. [14], but there are test case studies and has no relation to developing method.

Recently, many researchers increased their tendency towards meso-scale simulation. In this regard. Dissipative particle dynamics has been of great importance in mesoscale simulation of condensed matter especially for liquids [15]. This method is a mesh-less simulation method that is regarded as a serious alternative for molecular simulation [16]. Moreover, LBM as a mesh-based meso-scale approach method is believed to be an alternative for CFD. The LBM method has been used for studying different physical phenomena in Fluid Mechanics such as droplet dynamic [17], heat transfer [18] micro and nanoparticles suspensions [19] and porous media [20]. Main advantage of LBM over CFD is the simple structure and simplicity in coding. LBM methodology using BGK lattice model are linear equations and therefore easy and quick to solve, in contrast CFD method deals with solving a system of nonlinear equations simultaneously. This is because of the non-linear convective terms in Navier-Stokes equations which cause instability in reaching an explicit solution. Besides, CFD comes with various discretization schemes for pressure correction (such as SIMPLE or SIMPLEC algorithms), as well as solving the Laplace equation in every iteration of the implicit solution. All these aspects make the CFD more complicated to implement and computationally intense [21].

Agarwal [22] studied the effects of MHD on a conducting gas using LBM method. He stated that the LBM results had an acceptable agreement with the ones from analytical solutions. Chatterjee and Amiroudine [23] examined a case for an MHD micropump by LBM in which the results were close to the experimental and CFD ones. In another study by LBM, Kalteh and Abedinzadeh [24] analyzed the efficacy of magnetic field on nanofluids, which resulted the $86 \%$ increase of friction factor. In another study, effects of viscous dissipations on a magnetic micropump were studied by Jamalabadi and Park [25]. They found that the temperature profile near the wall increases by using the viscous dissipations effect. On the other hand, the temperature in the center line of the microchannel increased with the rise of the Joule heating. Despite all the aforementioned advantages of nanofluids and inverse magnetic field, one of its main drawbacks is increasing the shear stress. In order to overcome this problem, researchers use hydrophobic surfaces and also non-uniform magnetic field. This method is more advantageous in comparison with the uniform magnetic field in heat transfer applications. Adjusting magnetic field intensity along the surface of the heat transfer with two increasing or decreasing methods and also using a field with constant intensity for a fully developed flow are some of these advantages. In recent years, researchers have used the non-uniform magnetic field upon using LBM and CFD. In a numerical study, Aminifar et al. [26] examined the effect of linear magnetic field on flow behavior of a nanofluid. They stated that the implemented magnetic field performs better in lower Reynolds numbers. In a numerical study, Aminifar et al. [27] examined the heat transfer and friction factor of ferrofluid in which the geometry was a vertical duct. Finally, they simultaneously used both uniform and non-uniform magnetic fields. In another study, Aminifar et al. [28] analyzed flow and heat transfer in curved tubes under linear magnetic field. It was concluded that the secondary flow plays an important role in enhancing the heat transfer rate.

These studies mainly focused on investigating the effect of non-uniformity of magnetic field in enclosures. According to the authors, despite considering non-uniform magnetic fields, no previous researches have worked on improving the heat transfer in microchannels. The results of the present paper could lead to a pattern for the design of microchannels according to a special heat transfer characteristic. Moreover, this study tends to describe the thermal and friction behavior of alumina nanofluids in a microchannel. The surface of the microchannel has a constant temperature and it undergoes non-uniform magnetic field. Moreover, the surface has different degrees 
of hydrophobicity. To do so, the LBM equations are used along with variations to implemented hydrophobic surfaces and also source terms for considering non-uniform magnetic field. For this, the numerical process is first validated in several steps with experimental and numerical reported results. Next, a wide range of effective parameters are investigated.

Section 2 presents the details of simulated physical problem, where nanofluids formulation besides the IPLBM mathematics and after that boundary condition is discussed. Section 3 contains numerical procedure verification by explore the grid indepenct study and model validation. The result and discussion section involve the investigation of different parameter on heat transfer and hydrodynamic characteristics in microchannel (Sect. 4). Section 5 finally contains the overall findings are presented as conclusion.

\section{Problem description and simulation details}

The present study deals with the numerical simulation of the water/alumina nanofluid that flows in a rectangular 2D microchannel. The LBM method is used to simulate the flow. In this regard, a linearly decreasing magnetic field is implemented to the microchannel. The corresponding Hartmann number values are in the range of 0-40. Moreover, the presence of smooth walls in order to create sliding surfaces in the upper and lower walls of the microchannel for slip coefficient of 0-0.06 can be considered as important factors to increase heat transfer. The dimensions regarding the microchannel under study are of $L=1000$ $\mu \mathrm{m}$ and $\mathrm{H}=50 \mu \mathrm{m}$ for the length and height, respectively. Figure 1 illustrates the geometry of the problem.

In this study, the inlet temperature is $T_{\text {in }}=300 \mathrm{~K}$ and the temperature of the micro-channel walls is considered to be $T_{w}=320 \mathrm{~K}$. Moreover, the fluid enters the microchannel at constant velocity concerning the Reynolds number of $R e=50$. It should be noted that the heat transfer and flow characteristics of the microchannel are studied under the following circumstances: temperature jump and the slip velocity on the walls; consideration of the viscous dissipation and Joule heating; and finally the decreasing magnetic field.

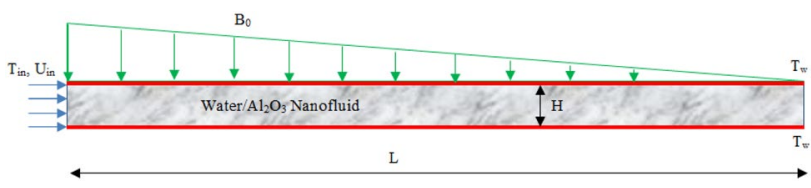

Fig. 1 The schematic of the examined geometry
Finally, the fluid regime is assumed to be in laminar regime, single-phase, incompressible, and Newtonian.

\subsection{Nanofluid properties}

In this study, the two phase mixture model is used to model the density and specific heat capacity [29].

$\rho_{n f}=\left(1-\varphi_{p}\right) \rho_{f}+\varphi_{p} \rho_{p}, \quad\left(\rho c_{p}\right)_{n f}=\left(1-\varphi_{p}\right)\left(\rho c_{p}\right)_{f}+\varphi_{p}\left(\rho c_{p}\right)_{p}$

Moreover, the effective viscosity [30] and effective thermal conductivity [31] of the nanofluid are calculated based on the following correlations:

$\frac{\mu_{n f}}{\mu_{f}}=\frac{1}{\left(1-1.35 \varphi_{p}\right)^{2.5}}, \quad \frac{k_{n f}-k_{f}}{k_{f}}=\frac{k_{p}}{k_{f}}\left(1+\right.$ CtePe $\left._{p}\right) \frac{d_{f}}{d_{p}} \frac{\varphi_{p}}{1-\varphi_{p}}$

In these correlations, $\mathrm{k}$ is the thermal conductivity and Cte is a constant number $(\mathrm{Cte}=25000)$. $\mathrm{Pe}_{p}$ stands for the Peclet number that is $P e_{p}=\frac{u_{p} d_{p}}{\alpha_{f}}$. Also, $u_{p}=\frac{2 k_{B} T}{\mu_{f} \pi d_{p}^{2}}$ is the nanoparticle brownian motion. Moreover, $\mathrm{T}$ and $\mathrm{K}_{B}$ are respectively the temperature of nanofluid and the Boltzmann constant. In addition, $\alpha_{f}$ represents the thermal diffusion coefficient of the base fluid. It should be stated that Table 1 shows the water and nanoparticles properties.

\subsection{Lattice Boltzmann method}

In the following, the discretized form of hydrodynamic Boltzmann equation is given which consists of two collision and streaming steps. The density distribution function in a discretized form is as follows [34]:

$f_{k}(r+\Delta r, t+\Delta t)-f_{k}(r, t)=-\frac{\Delta t}{\tau_{f}}\left[f_{k}(r, t)-f_{k}^{e q}(r, t)\right]+\Delta t F_{k}$

Moreover, the kinematic viscosity and relaxation time of density distribution function are [19]:

$v=\gamma C_{s}^{2}\left(\frac{\tau_{f}}{\Delta t}-0.5\right) \Delta t, \quad C_{s}=\frac{\frac{\Delta r}{\Delta t}}{\sqrt{3}}, \quad r=x \boldsymbol{i}+y \boldsymbol{j}$.

where $0<\gamma \leq 1$ is a parameter that can be arbitrarily chosen, and $c_{s}=\Delta x /(\sqrt{3} \Delta t)$ is the speed of sound. Moreo-

Table 1 Properties of water [32] and Alumina nanoparticles [33]

\begin{tabular}{lllll}
\hline & $\rho\left(\mathrm{kg} / \mathrm{m}^{3}\right)$ & $\mathrm{Cp}(\mathrm{J} / \mathrm{kg} \mathrm{K})$ & $\mathrm{k}(\mathrm{W} / \mathrm{m} \mathrm{K})$ & $\mu(\mathrm{Pa} \mathrm{s})$ \\
\hline Water & 996.16 & 4178 & 0.605 & 0.00085 \\
Alumina & 3971 & 779 & 40 & - \\
\hline
\end{tabular}


ver, the source term regarding the MHD in LBM is in the following form:

$F_{k}=w_{k}\left(1-\frac{\Delta t}{2 \tau_{f}}\right)\left[\frac{\boldsymbol{C}_{\boldsymbol{k}}-\boldsymbol{V}}{c_{s}^{2}}+\left(\frac{\boldsymbol{C}_{\boldsymbol{k}} \cdot \boldsymbol{V}}{c_{s}^{4}}\right) c_{k}\right] \cdot \rho \boldsymbol{a}$

where $\vec{a}$ denotes the acceleration that is related to the magnetic field:

$\overrightarrow{\mathrm{a}}=a x \boldsymbol{i}+a y \boldsymbol{j}=-\frac{B_{0}^{2} \sigma_{n f}}{\rho} \boldsymbol{i}+0 \boldsymbol{j}$

In which, $\mathrm{B}_{0}^{2} \sigma_{\mathrm{nf}}=\frac{\mathrm{Ha}^{2} \mu_{\mathrm{nf}}}{\mathrm{H}^{2}}$. Where $\sigma_{\mathrm{nf}}$ is the nanofluid electric current and Ha stands for the Hartmann number. The microscopic velocity vectors in LBM are defined as:

$c= \begin{cases}(0,0) & k=0 \\ \left(\cos \frac{\pi(k-1)}{2}, \sin \frac{\pi(k-1)}{2}\right) \frac{\Delta x}{\Delta t} & k=1-4 \\ \sqrt{2}\left(\cos \frac{\pi(2 k-1)}{4}, \sin \frac{\pi(2 k-1)}{4}\right) \frac{\Delta x}{\Delta t} & k=5-8\end{cases}$

Finally, we have the density distribution function, which is in the following form:

$\mathrm{f}_{\mathrm{k}}^{\mathrm{eq}}=\rho w_{k}\left[1+\frac{\boldsymbol{C}_{\boldsymbol{k}} \cdot \mathbf{V}}{c_{s}^{2}}+\frac{1}{2}\left(\frac{\boldsymbol{C}_{\boldsymbol{k}} \cdot \mathbf{V}}{c_{s}^{2}}\right)^{2}-\frac{\mathbf{V} \cdot \mathbf{V}}{2 c_{s}^{2}}\right]$

In recent equation, $\mathrm{w}_{\mathrm{k}}$ is the weighting function.

$W_{k}=\left\{\begin{array}{ll}\frac{1}{4} & k=0 \\ \frac{1}{9} & k=1-4 \\ \frac{1}{36} & k=5-8\end{array}\right\}$

Equation 10 states an equation for the temperature distribution function having a source term which is as follows [35]:

$\frac{g_{k}(r+\Delta r, t+\Delta t)-g_{k}(r, t)}{\Delta t}=-\frac{1}{\tau_{g}}\left[g_{k}(r, t)-g_{k}^{e q}(r, t)\right]+S_{k}$

where:

$S_{k}=w_{k}\left[1+\frac{\left(\boldsymbol{C}_{\boldsymbol{k}} \cdot \mathbf{V}\right)}{C_{s}^{2}}\left(\frac{\tau_{g}-0.5 \Delta t}{\tau_{g}}\right)\right] S$

where $S_{k}$ is the source term along the lattice links. Also, $S$ represents a source term caused by the viscous dissipations [36],

$S=\frac{\mu_{n f}}{\left(\rho c_{p}\right)_{n f}}\left\{2\left[\left(\frac{\partial u}{\partial x}\right)^{2}+\left(\frac{\partial v}{\partial y}\right)^{2}\right]+\left(\frac{\partial u}{\partial y}+\frac{\partial v}{\partial x}\right)^{2}\right\}$

The thermal diffusion coefficient is expressed as:
$\alpha=C_{s}^{2}\left(\frac{\tau_{g}}{\Delta t}-0.5\right) \Delta t$

In this regard, the temperature distribution function is based on the following relation:

$g_{k}^{e q}=w_{k} T\left[1+\left(\frac{\boldsymbol{C}_{\boldsymbol{k}} \cdot \mathbf{V}}{c_{s}^{2}}+\frac{1}{2}\left(\frac{\boldsymbol{C}_{\boldsymbol{k}} \cdot \mathbf{V}}{c_{s}^{2}}\right)^{2}-\frac{\mathbf{V} \cdot \mathbf{V}}{2 c_{s}^{2}}\right)\right]$

Finally, Eq. 15 presents the macroscopic density, velocity, and temperature:

$\left\{\begin{array}{l}\rho=\sum_{k=0}^{8} f_{k} \\ \rho V=\sum_{k=0}^{8} \boldsymbol{C}_{\boldsymbol{k}} \cdot f_{k}+\frac{\Delta t}{2} \rho a \\ T=\sum_{k=0}^{8} g_{k}\end{array}\right.$

\subsection{Boundary conditions}

The fluid enters the microchannel upon constant velocity and temperature [37]. Concerning the outlet boundary condition, the distribution functions are extrapolated [38]. Moreover, according to the study by [8], the surface hydrophobicity condition has been applied for the microchannel surface:

$u=\left.B \frac{\partial u}{\partial Y^{*}}\right|_{Y^{*}=0}$

where $B=\frac{\beta}{H}$ represents the dimensionless slip coefficient. $\beta$ is the slip parameter and $Y^{*}$ is a dimensionless parameter related to the vertical axis. Also, the velocity slip at the surface is:

$u_{0}=\frac{B}{2 \Delta Y^{*}+B}\left(4 u_{1}-u_{2}\right)$

Upon using the method in [39], the unknown distribution functions are based on the following form:

$\left\{\begin{array}{l}f_{2}=f_{4} \\ f_{5}=\frac{\left(\rho_{w}+\rho_{0} u_{0}\right)-\left(f_{0}+f_{2}+f_{4}\right)-0.5 \rho a x u_{0}}{2}-\left(f_{1}+f_{8}\right) \\ f_{6}=\frac{\left(\rho_{w}-\rho_{0} u_{0}\right)-\left(f_{0}+f_{2}+f_{4}\right)+0.5 \text { paxu }}{2}-\left(f_{3}+f_{7}\right)\end{array}\right.$

As the microchannel is symmetrical, we simply use the symmetry boundary condition [37]: Also, in order to implement the temperature jump in the upper wall, we used [40]: 
$T_{n f}=T_{w}+\zeta^{*} \frac{\partial T}{\partial y^{*}}{ }_{\mid y^{*}=1}$

In which $\zeta^{*}$ is the dimensionless coefficient representing the temperature jump which is as follows:

$\zeta^{*}=\frac{\zeta}{H}=\frac{B 2 \gamma}{\operatorname{Pr}(\gamma+1)}$

In the above relation, $\zeta$ is the dimensionlal temperature jump coefficient and $\gamma$ is the relative specific heat ratio which is usually taken as 1 for liquids. The dimensionless parameters in the present study are expressed as follows:
$(\mathrm{Nu})$ and coefficient of friction $\left(C_{f}\right)$ are studied based on Table 2. According to the Fig. 2 and Table 2, the grid with a resolution of $70 \times 1400$ has been selected for further simulations.

In order to perform the validation process, the velocity profile has been compared with the analytical solution presented by $[8,13]$ (Fig. 3-a). Moreover, concerning the fluid with $\operatorname{Pr}=0.7$ and $\gamma=1.4$, the dimensionless temperature profile at the outlet was compared with the analytical solution of the [41] (Fig. 3-b). Also, in order to validate the source term in the temperature equation, the air Nusselt number with $\mathrm{Pr}=0.7$ has been compared with the analyti-

$X^{*}=\frac{x}{H} \quad Y^{*}=\frac{y}{H} \quad u^{*}=\frac{u}{u_{i n}} \quad v^{*}=\frac{v}{u_{i n}} \quad \theta=\frac{T-T_{\text {in }}}{\frac{q^{\prime \prime} H}{k_{f}}} \quad \theta_{F D}=\frac{T-T_{w}}{\frac{q^{\prime \prime} H}{2 k_{f}}}$,

$\overline{\tau_{w}}=\frac{\left.\int_{0}^{L} \mu_{n f} \frac{\partial u}{\partial y}\right|_{y=0} d x}{L}, \quad \overline{c_{f}}=\frac{2 \overline{\tau_{w}}}{\rho u_{i n}^{2}}, \quad N u=\frac{-\left.k_{n f} \frac{\partial T}{\partial y}\right|_{y=H c}}{T_{w}-T_{i n}} \frac{H c}{k_{f}}, \quad \overline{N u}=\frac{\int_{0}^{L} N u d x}{L}$

\section{Grid Independence and validation}

In this section, we present the grid independence check and the validation. As observed in Fig. 2. It can be seen that the local friction factor of nanofluid along the microchannel is shown, which includes the temperature jump on the microchannel walls and also the viscous dissipation. Moreover, these data correspond to the following dimensionless numbers: $\mathrm{Ha}=30, \mathrm{Re}=50$ and $\mathrm{B}=0.1$. Concerning the grid independence check, the average Nusselt number

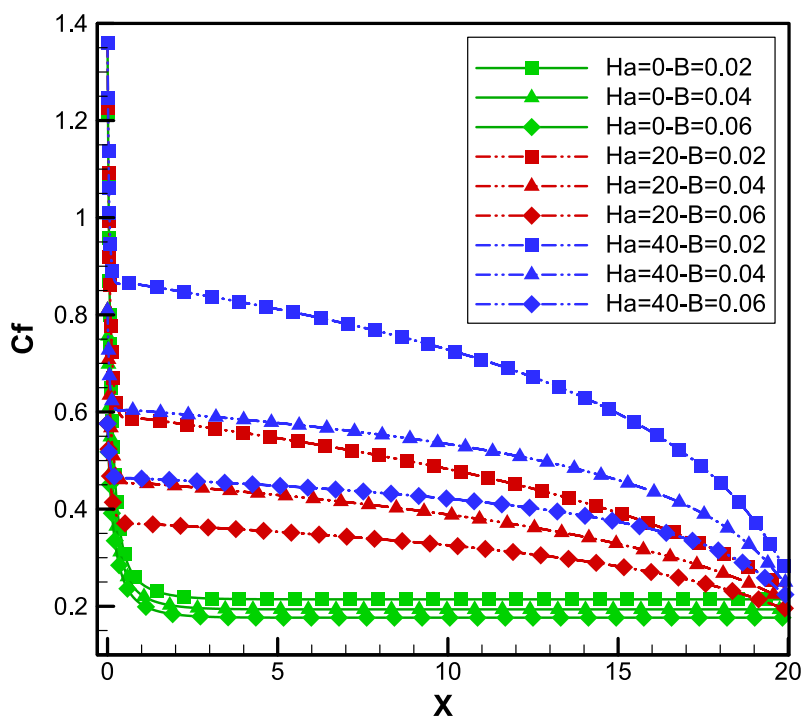

Fig. 2 Local friction factor changes along the bottom of the microchannel for different Hartmann numbers and slip coefficients at pho $=2 \%$ cal solution in [19]. The comparison is carried out for different Brinkman numbers and it can be observed in Table 3.

Table 2 Average Nusselt number and friction factor for $\mathrm{Ha}=30$, $\operatorname{Re}=50$ and $B=0.1$

\begin{tabular}{lcccc}
\hline$n \times m$ & $30 * 600$ & $50 * 1000$ & $70 * 1400$ & $90 * 1800$ \\
$\overline{\overline{C f}}$ & 0.23 & 0.24 & 0.24 & 0.24 \\
$\overline{\mathrm{Nu}}$ & 15.66 & 15.7 & 15.7 & 15.7 \\
\hline
\end{tabular}

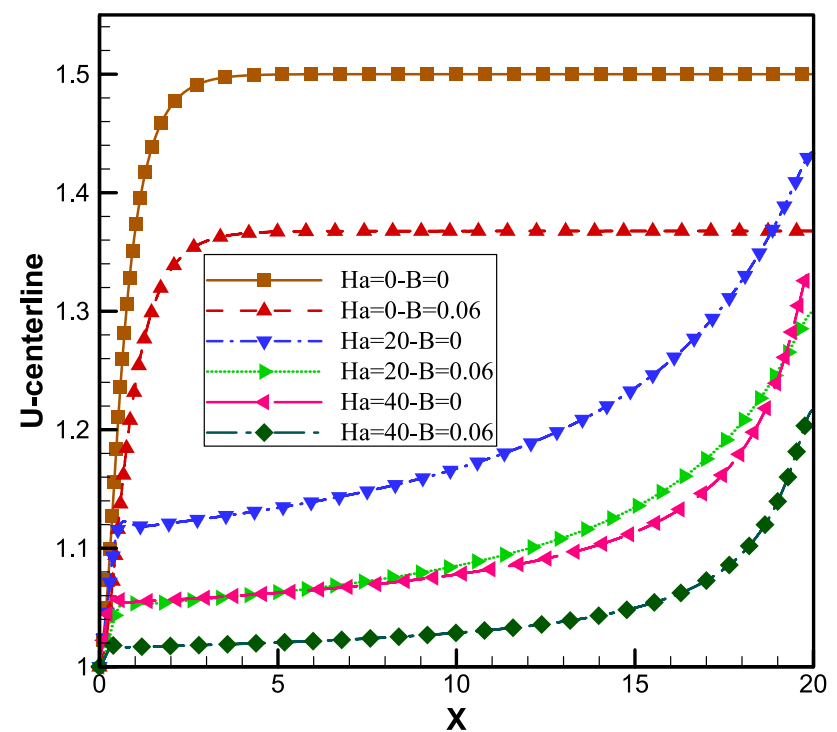

Fig. 3 Dimensionless velocity changes in the $x$ direction along the microchannel for different Hartmann numbers and slip coefficients at $\mathrm{phi}=2 \%$ 
Table 3 Nusselt number comparison between present study and the analytical solution for different Brinkman numbers and $\operatorname{Pr}=0.7$

\begin{tabular}{llllr}
\hline $\mathrm{Br}$ & 0.001 & 0.01 & 0.1 & \multicolumn{1}{c}{1} \\
\hline $\mathrm{Nu}_{\mathrm{FD}}$ (present work) & 5.38 & 5.28 & 4.45 & 1.73 \\
$\mathrm{Nu}_{\mathrm{FD}}$ analytical [19] & 5.37 & 5.27 & 4.46 & 1.75 \\
Error (relative) (\%) & 0.13 & 0.11 & -0.13 & -0.91 \\
\hline
\end{tabular}

The validation continues for implementing the temperature jump and the velocity slip, in which the average Nusselt number is compared with the study done by Karimipour et al. in $R e=100, H a=0,20,40$ and $B=0.01$ for alumina-water nanofluid with volume concentration of $4 \%$ [11] in different Hartmann numbers (Table 4).

After having performed these processes, we finally compared the Nusselt number with the ones from the experimental results. The microchannel of interest consists of the height and length of $200 \mu \mathrm{m}$ and $50 \mathrm{~mm}$, respectively. Also, the volume concentration of the nanofluid was 2 percent (Titanium with water as the base fluid). Finally, the mass flow rate of the fluid was $37.7 \mathrm{~kg} / \mathrm{h}$. Table 5 shows the mentioned comparison.

\section{Results and discussion}

In this study, the flow characteristics of water-alumina nanofluid in a microchannel are investigated for the Reynolds number ranges from 50 to 300 , the volume fraction of nanoparticles from 0 to $2 \%$, the Hartmann number from 0 to 40 and the dimensionless slip coefficient from 0 to 40 . The constant temperature of the microchannel surface is $320 \mathrm{~K}$. The velocity slip, temperature jump and viscous dissipation terms are incorporated in all results unless where indicated not to be included.

\subsection{Local friction factor}

In Fig. 2, the local friction factor variations along the bottom of the microchannel wall for Hartmann numbers 0-40 and the slip coefficient $0-0.06$ has been compared. In these diagrams, the effect of increasing wall slip and magnetic

Table 4 Result comparison of the present work with Karimipour et al. [11]

\begin{tabular}{lccc}
\hline Averaged Nusselt & \multicolumn{4}{l}{ Hartman number } \\
\cline { 2 - 4 } & 0 & 20 & 40 \\
\hline Present work & 4.46 & 5.1 & 5.33 \\
Karimipour et al. [11] & 4.5 & 5.12 & 5.28 \\
Relative deviation (\%) & -0.82 & -0.33 & 0.97 \\
\hline
\end{tabular}

Table 5 Nusselt number comparison of the present work and the experimental investigation [42]

\begin{tabular}{lrrr}
\hline$x(\mathrm{~m})$ & 0.007 & 0.027 & 0.047 \\
\hline Pure water & & & \\
$\quad$ Nusselt number (present work) & 9.33 & 6.47 & 5.81 \\
$\quad$ Nusselt number [42] & 10.21 & 6.76 & 5.47 \\
$\quad$ Error (relative) (\%) & -8.62 & -4.29 & 6.22 \\
$\mathrm{TiO}_{2}$-water 2\% & & & \\
Nusselt number (present work) & 9.19 & 6.34 & 5.70 \\
Nusselt number [42] & 9.67 & 6.42 & 5.24 \\
Error (relative) (\%) & -4.96 & -1.25 & 8.78 \\
\hline
\end{tabular}

field intensity on skin friction factor variations are shown. As the slip increases, the level of solid surface resistance against fluid motion decreases and the fluid flow will have less momentum drops, and the cooling fluid will travel the path along the microchannel with less momentum drop. On the other hand, increase of the Lorentz force intensity with the consequent increase of the magnetic field leads to increase of the fluid adherence to the solid wall, which will increase the skin friction factor. In addition to approaching the hydrodynamic development of the flow behavior, the presence of a linear-decreasing magnetic field can also bring about the declining trend of local friction factor, which at the end of the microchannel causes a steep slope in these graphs, especially for higher field intensities. Also, increasing the slip velocity coefficient causes proper behavior and decrease of the local friction factor along the fluid flow direction. Thus, by polishing the microchannel surface, better contact with solid surfaces in higher Hartmann numbers can be associated with a lower friction factor. This behavior is completely apparent with the interference of friction factors in Hartmann numbers 40 and 20 with higher slip coefficient.

\subsection{Investigating the behavior of dimensionless velocity changes in the central flow line}

In Fig. 3, the graphs of the velocity changes in the direction of fluid motion along the microchannel for Hartmann numbers 0,20 and 40 , and the slip coefficient $B=0$ and $B=0.06$ are shown in the central line of the microchannel. Due to changes in the axial velocity diagrams, the application of the magnetic field affects the velocity field. As the flow is not affected by the magnetic field, axial velocity has increased due to the formation of the boundary layer and the no-slip boundary condition in the microchannel walls. The reason for this behavior is the flow continuity and the formation of inviscid core in the farthest areas from the wall and in the central line of the flow. The decrease of the passage surface in the viscous region due to the 
formation of the velocity boundary layer has increased the fluid velocity in the center of the microchannel, which has the axial velocity alignment. As a result, with increasing slip velocity in all of the studied cases, the level of axial velocity graphs has decreased. The application of a linear magnetic field to a cooling fluid causes changes in Lorentz force and its effects on flow behavior. By moving the fluid to the end of the microchannel, the effects of linear Lorentz force have decreased and the velocity gradients have increased in the fluid layers. This increases the slip between the fluid layers and, consequently, the formation of a boundary layer with the progression of the fluid to the end of the microchannel. Therefore, the flow velocity has increased due to the flow continuity in the microchannel and the alignment of the axial velocity diagrams has increased, consequently.

\subsection{Analysis of the dimensionless temperature profile}

Figure 4 investigates the variations of the dimensionless temperature profiles in two sections $X=0.1$ and $X=0.9$ for the Hartmann numbers 0 and 40 , and the slip coefficient $B=0$ and $B=0.06$. In these diagrams, the effect of intensity increase of the magnetic field and the slip velocity coefficient in the vertical direction of the flow in the initial and the end sections of the microchannel is investigated. At the beginning of the microchannel, due to the type of linear profile, the flow field was mostly influenced by the magnetic field and the temperature gradients were less in the fluid layers, and even the increase of the slip velocity on the solid surface did not have much effect on the changes in the temperature profile. It seems that the movement of a bulk of fluid can be due to the lack of growth of temperature gradients and the approaching behavior of the temperature profiles at the beginning of the microchannel. In such a way that even slip velocities cannot lead to changes in the temperature between the fluid layers at the beginning of the microchannel. With a higher fluid flow, the effect of the field intensity is reduced and the effects of temperature gradients can differentiate the temperature profiles in the microchannel end regions. Slip velocity coefficient variations in these areas also differentiate behavior in the temperature graphs. The increase in this coefficient facilitates the fluid flow and stability of the inlet temperature over longer than the fluid motion, and the effects of the thermal boundary layer penetration in the central region of the stream flow. Hence, the dimensionless temperature graph in Fig. 4 is broader for $B=0.06$ and the regions with lower temperatures cover more vertical sections of the microchannel.

Figure 5 demonstrates the variations of the dimensionless temperature profiles in three sections for $\mathrm{X}=0.2$,

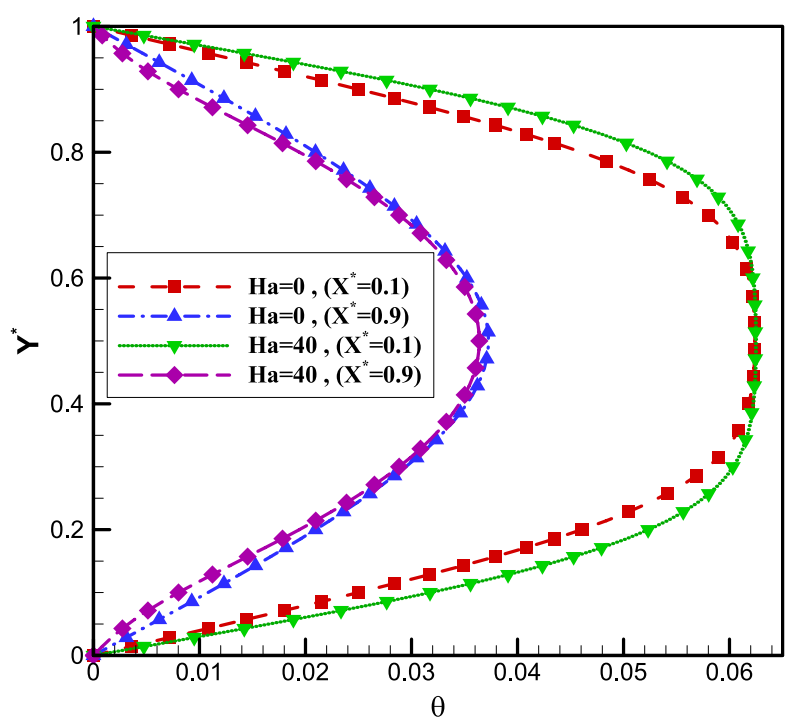

A) $B=0$

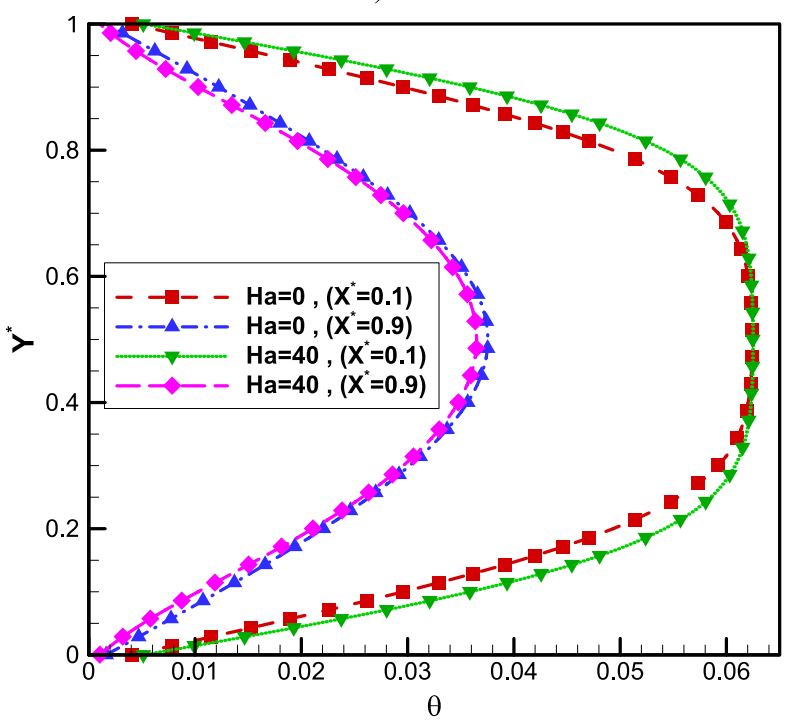

B) $\mathrm{B}=0.06$

Fig. 4 Changes of the dimensionless temperature profile in two sections $X=0.1$ and $X=0.9$ for different Hartmann numbers and slip coefficients of 0 (a) and 0.06 (b)

$X=0.5,=0.8$ for different Hartmann numbers and slip coefficients. With the advancement of fluid in the microchannel, due to the heat absorption from high temperature walls, the temperature of the cooling fluid increases and the heat transfer rate is reduced. Factors such as increasing the slip velocity in the microchannel walls reduce the improvement of the temperature distribution in layers above the fluidized surface and reduce the temperature gradients. Therefore, the temperature profiles are closer to the inlet fluid temperature by decreasing the fluid flow and increasing the slip velocity, and the inlet temperature stability is improved. 


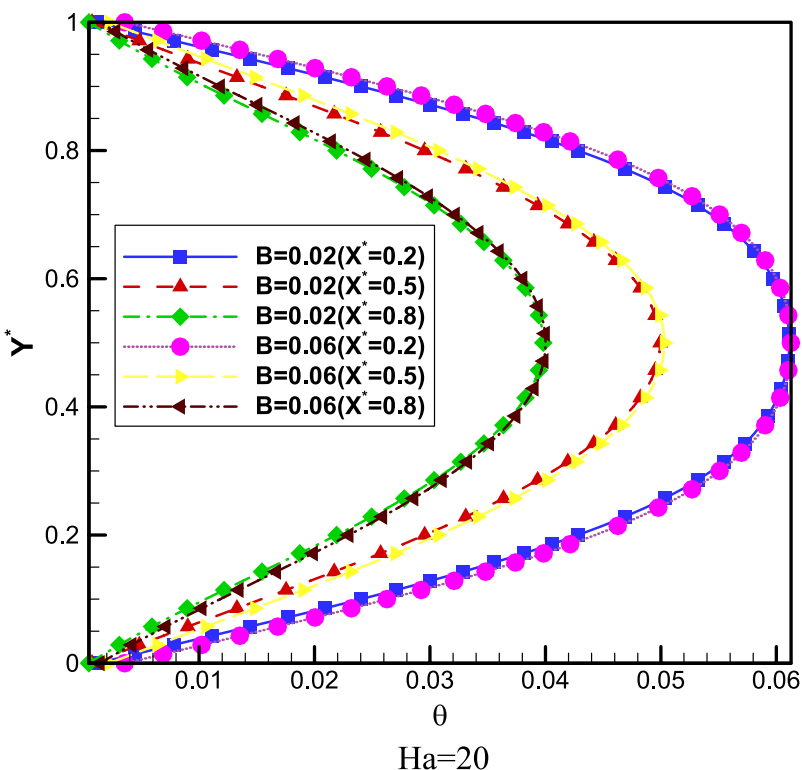

Fig. 5 Changes of the dimensionless temperature profile in three sections $X=0.2, X=0.5$ and $X=0.8$ for different Hartmann numbers and slip coefficients

\subsection{Average Nusselt numbers graphs}

In Figs. 6 and 7, the average Nusselt number variations are compared with the change of the dimensionless slip coefficient (B) in different Hartmann numbers $\mathrm{(Ha}$ ) at two concentrations of $0 \%$ and $2 \%$. Graphs show that the nanofluid increases the heat transfer rate about 1.3 times for all conditions. So that, the process of changing the Nusselt number for a nanofluid with a $2 \%$ volume fraction of nanoparticles is remarkable compared to the base fluid. The slip presence in the walls of the microchannel has also boosted the flow of the fluid and reduced velocity gradients due to the presence of solid surfaces. Thus, upon increasing the slip velocity coefficient, the fluid velocity is stable, and the heat transfer coefficient and Nusselt number are strengthened. The application of a linear magnetic field also results in better fluid adherence to the surface and better absorption of heat by a fluid passing through the microchannel, which is also evident in the temperature diagrams.

\subsection{Average shear stress on the bottom solid wall}

In Fig. 8, the average shear stress variation with respect to the dimensionless slip coefficient (B) in different Hartmann numbers in two different volume fraction of nanoparticles is shown. In this section, the effect of Hartmann number and slip velocity coefficient, and the presence of solid nanoparticles with volume fraction of nanoparticles $2 \%$ on the solid wall shear stress behavior are compared, simultaneously. The fluid motion on the solid wall and its contact with the fluid

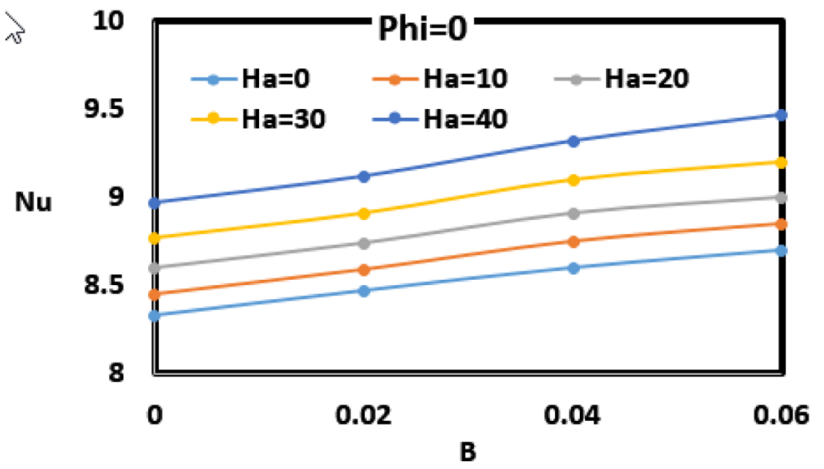

a. $\varphi=0$

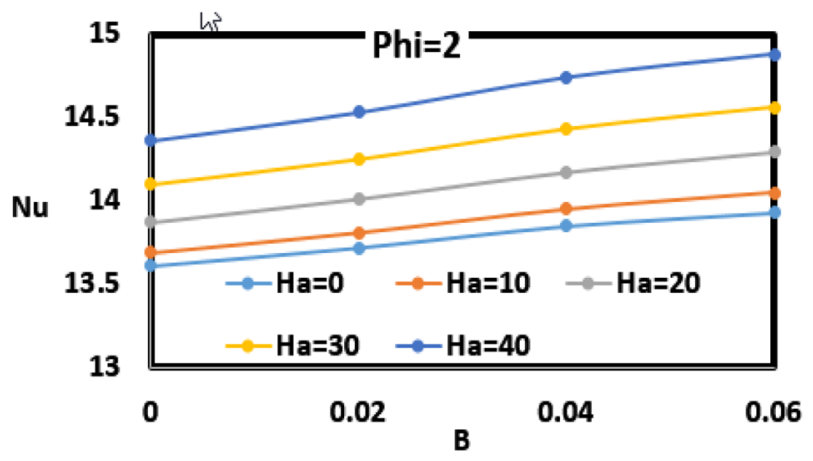

b. $\varphi=0.02$

Fig. 6 the average Nusselt number versus dimensionless slip coefficient $(\mathrm{B})$ in different Hartmann numbers $(\mathrm{Ha})$ at two concentrations of $0 \%$ and $2 \%$

can lead to changes in the velocity components of the fluid and the friction factor and ultimately the shear stress. The presence of solid nanoparticles increases the shear stresses due to increased viscosity and coolant fluid density. On the other hand, by increasing the slip velocity, fluid and solid contact on the walls of the microchannel is less felt by the fluid and the smooth surface will produce less friction and ultimately lower shear stress. The application of Lorentz force is due to the magnetic field existence and an increase in the intensity of the field. The fluid is tightly attached to the surface and fully contacts it, which causes the component to reduce the component of the axial velocity due to the higher wear and tear in the fluid passing through the solid surfaces. For this reason, the shear stress increases with the increase of the magnetic.

\section{Conclusion}

The present study has analyzed the water/alumina nanofluids in a rectangular microchannel. A linearly decreasing magnetic field is implemented over the microchannel for 


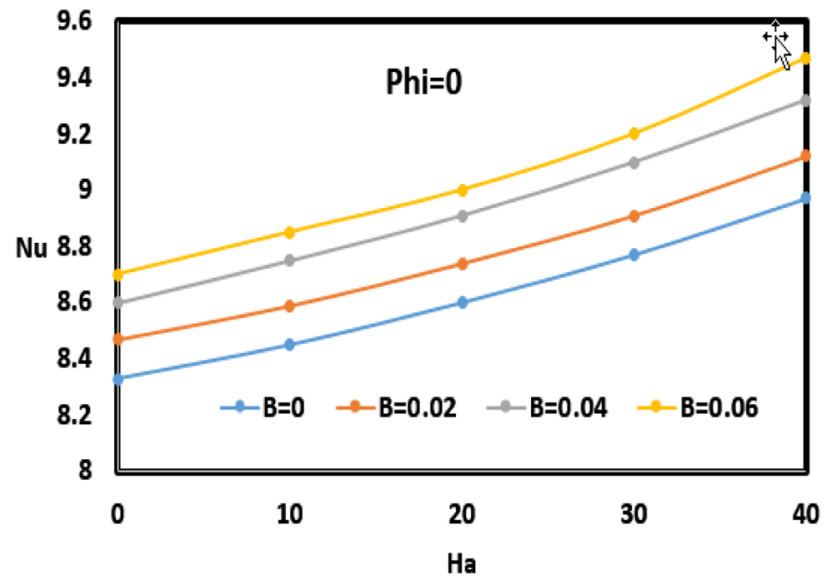

a. $\varphi=0$

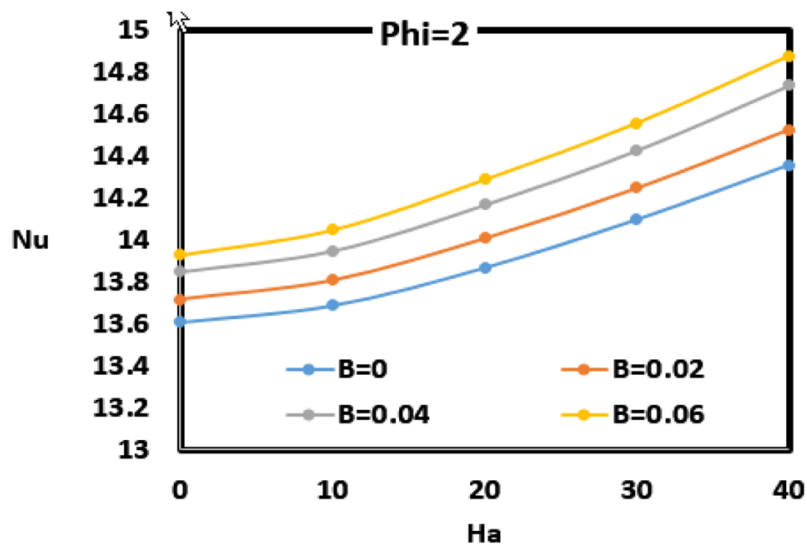

b. $\varphi=0.02$

Fig. 7 the average Nusselt number versus Hartmann numbers $(\mathrm{Ha})$ for different dimensionless slip coefficient (B) at two concentrations of $0 \%$ and $2 \%$

Hartmann numbers of $0,10,20,30$ and 40 . The simulation of this research was carried out using LBM in a twodimensional space. The results of this study indicate that the increase in the magnetic field due to the increase of Lorentz force intensity to the cooling fluid, leads to greater fluid adherence to the solid wall, which will increase the friction factor. Increasing the slip velocity coefficient causes proper behavior and reduces the local friction factor along the fluid flow path. Hence, by smoothing the microchannel surface, better contact with solid surfaces in higher Hartmann numbers can be obtained with a lower friction factor. As the slip velocity coefficient increases, the effects of the presence of solid walls and the formation of the boundary layer in these regions are reduced and the fluid will travel with less inhibition. As a result, with increasing slip velocity in all of the studied cases, the level of axial velocity graphs has decreased. By increasing the solid surface slip velocity, the fluid velocity is stable, and the heat transfer coefficient and Nusselt number are

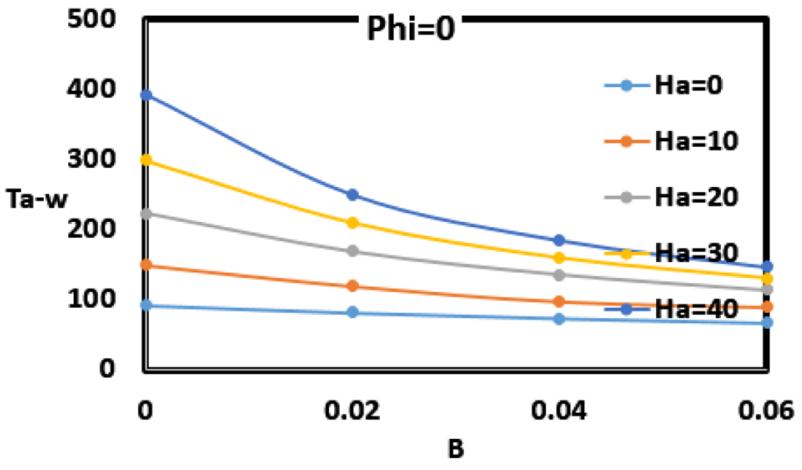

a. $\varphi=0$

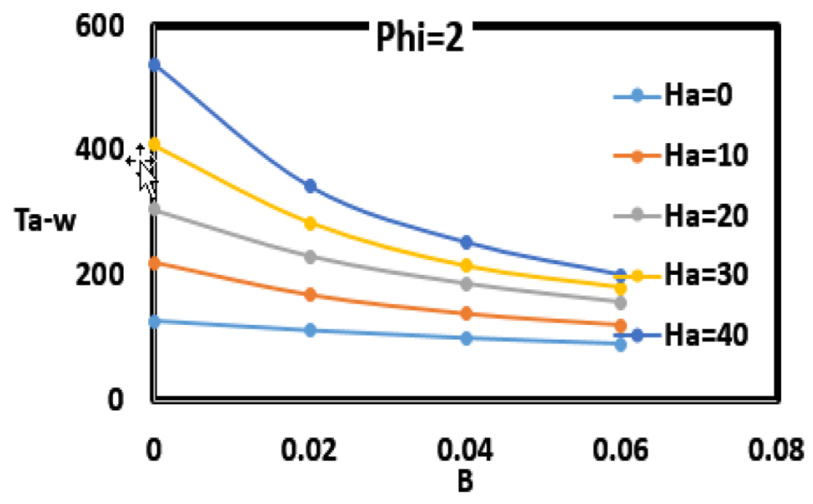

b. $\varphi=0.02$

Fig. 8 the average shear stress versus dimensionless slip coefficient (B) in different Hartmann numbers in two different volume fractions of nanoparticles

strengthened. The application of a linear magnetic field also results in better fluid adherence to the surface and better absorption of heat by the fluid passing through the microchannel, which is also evident in the temperature diagrams. The application of Lorentz force is due to the presence of a magnetic field and an increase in the intensity of the field. The fluid is tightly attached to the surface and fully contacts it, which causes reduction in the component of the axial velocity due to the higher wear and tear in the fluid passing through the solid surfaces.

\section{Compliance with ethical standards}

Conflict of interest The authors declare that they have no conflict of interest.

\section{References}

1. Akbari $O A$, Goodarzi $M$, Safaei $M R$, Zarringhalam $M$, Ahmadi Sheikh G, Shabani R, Dahari M (2016) A modified two-phase mixture model of nanofluid flow and heat transfer in 3-d 
curved microtube. Adv Powd Technol 27:2175-2185. https:// doi.org/10.1016/j.apt.2016.08.002

2. Chaurasia P, Kumar A, Yadav A, Rai PK, Kumar V, Prasad L (2019) Heat transfer augmentation in automobile radiator using $\mathrm{Al}_{2} \mathrm{O}_{3}$-water based nanofluid. SN Appl Sci 1(3):257. https://doi. org/10.1007/s42452-019-0260-7

3. Sheikholeslami M (2019) New computational approach for exergy and entropy analysis of nanofluid under the impact of Lorentz force through a porous media. Comput Methods Appl Mech Eng 344:319-333. https://doi.org/10.1016/j. cma.2018.09.044

4. Aminossadati S, Raisi A, Ghasemi B (2011) Effects of magnetic field on nanofluid forced convection in a partially heated microchannel. Int J Non-Linear Mech 46(10):1373-1382. https://doi. org/10.1016/j.ijnonlinmec.2011.07.013

5. Morini GL (2005) Viscous heating in liquid flows in microchannels. Int J Heat Mass Transf 48(17):3637-3647. https://doi. org/10.1016/j.ijheatmasstransfer.2005.01.011

6. Hung YM (2010) Analytical study on forced convection of nanofluids with viscous dissipation in microchannels. Heat Transfer Eng 31(14):1184-1192. https://doi.org/10.1080/0145763100 3689344

7. Azad A, Rahman M, Öztop HF (2014) Effects of joule heating on magnetic field inside a channel along with a cavity. Procedia Eng 90:389-396. https://doi.org/10.1016/j.proeng.2014.11.867

8. Tretheway DC, Meinhart CD (2002) Apparent fluid slip at hydrophobic microchannel walls. Phys Fluids 14(3):L9-L12. https:// doi.org/10.1063/1.1432696

9. Akbarinia A, Abdolzadeh M, Laur R (2011) Critical investigation of heat transfer enhancement using nanofluids in microchannels with slip and non-slip flow regimes. Appl Therm Eng 31(4):556-565. https://doi.org/10.1016/j.appltherma leng.2010.10.017

10. Karimipour A, Afrand M (2016) Magnetic field effects on the slip velocity and temperature jump of nanofluid forced convection in a microchannel. Proc Inst Mech Eng Part C J Mech Eng Sci 230(11):1921-1923. https://doi.org/10.1177/095440621558623 2

11. Karimipour A, D'Orazio A, Shadloo MS (2017) The effects of different nano particles of $\mathrm{Al} 2 \mathrm{O} 3$ and $\mathrm{Ag}$ on the MHD nano fluid flow and heat transfer in a microchannel including slip velocity and temperature jump. Physica E 86:146-153. https://doi. org/10.1016/j.physe.2016.10.015

12. Abbaszadeh M, Ababaei A, Arani AAA, Sharifabadi AA (2017) MHD forced convection and entropy generation of $\mathrm{CuO}$-water nanofluid in a microchannel considering slip velocity and temperature jump. J Braz Soc Mech Sci Eng 39(3):775-790. https:// doi.org/10.1007/s40430-016-0578-7

13. Hamdan MA, Al-Assaf AH, Al-Nimr MA (2016) The effect of slip velocity and temperature jump on the hydrodynamic and thermal behaviors of MHD forced convection flows in horizontal microchannels. Iran J Sci Technol Trans Mech Eng 40(2):95-103. https://doi.org/10.1007/s40997-016-0004-x

14. Akbari OA, Karimipour A, Toghraie Semiromi D, Safaei MR, Alipour H, Goodarzi M, Dahari M (2016) Investigation of Rib's height effect on heat transfer and flow parameters of laminar water- $\mathrm{Al}_{2} \mathrm{O}_{3}$ nanofluid in a two dimensional rib-microchannel. Appl Math Comput 290:135-153. https://doi.org/10.1016/j. amc.2016.05.053

15. Afrouzi $\mathrm{HH}$, Moshfegh A, Javadzadegan A, Mohammadi M, Farhadi M (2018) Systematic fine-tuning of dissipative particle dynamics for mesoscale semidilute and dense colloidal suspensions. Phys A 510:492-506. https://doi.org/10.1016/j.physa 2018.07.005

16. Sharifian A, Moshfegh A, Javadzadegan A, Hassanzadeh Afrouzi H, Baghani M, Baniassadi M (2019) Hydrogenation-controlled mechanical properties in graphene helicoids: exceptional distribution-dependent behavior. Phys Chemi Chem Phys 21:1242312433. https://doi.org/10.1039/C9CP01361J

17. Tilehboni SM, Fattahi E, Afrouzi HH, Farhadi M (2015) Numerical simulation of droplet detachment from solid walls under gravity force using lattice Boltzmann method. J Mol Liq 212:544-556. https://doi.org/10.1016/j.molliq.2015.10.007

18. Nazari M, Ramzani S (2014) Cooling of an electronic board situated in various configurations inside an enclosure: lattice Boltzmann method. Meccanica 49(3):645-658. https://doi. org/10.1007/s11012-013-9817-5

19. Afrouzi $H H$, Sedighi K, Farhadi M, Moshfegh A (2015) Lattice Boltzmann analysis of micro-particles transport in pulsating obstructed channel flow. Comput Math Appl 70(5):1136-1151. https://doi.org/10.1016/j.camwa.2015.07.008

20. Sheikholeslami M (2018) CuO-water nanofluid flow due to magnetic field inside a porous media considering Brownian motion. J Mol Liq 249:921-929. https://doi.org/10.1016/j.molli q.2017.11.118

21. Mehrizi AA, Farhadi M, Shayamehr S (2013) Natural convection flow of $\mathrm{Cu}$-Water nanofluid in horizontal cylindrical annuli with inner triangular cylinder using lattice Boltzmann method. Int Commun Heat Mass Transfer 44:147-156. https://doi. org/10.1016/j.icheatmasstransfer.2013.03.001

22. Agrawal R (2003) Lattice Boltzmann simulation of magnetohydrodynamic slip flow in microchannels. Bull Am Phys Soc 48(10):93. https://doi.org/10.1016/B978-044452024-1/50034-8

23. Chatterjee $D$, Amiroudine S (2011) Lattice Boltzmann simulation of thermofluidic transport phenomena in a DC magnetohydrodynamic (MHD) micropump. Biomed Microdevice 13(1):147157. https://doi.org/10.1007/s10544-010-9480-8

24. Kalteh $M$, Abedinzadeh SS (2018) Numerical investigation of MHD nanofluid forced convection in a microchannel using lattice Boltzmann method. Iran J Sci Technol Trans Mech Eng 42:23-34. https://doi.org/10.1007/s40997-017-0073-5

25. Jamalabadi MA, Park JH (2014) 2014 Thermal radiation, joule heating, and viscous dissipation effects on MHD forced convection flow with uniform surface temperature. Open J Fluid Dyn 4(2):125. https://doi.org/10.4236/ojfd.2014.42011

26. Aminfar H, Mohammadpourfard M, Kahnamouei YN (2011) A 3D numerical simulation of mixed convection of a magnetic nanofluid in the presence of non-uniform magnetic field in a vertical tube using two phase mixture model. J Magn Magn Mater 323(15):1963-1972. https://doi.org/10.1016/j. jmmm.2011.02.039

27. Aminfar H, Mohammadpourfard M, Mohseni F (2012) Twophase mixture model simulation of the hydro-thermal behavior of an electrical conductive ferrofluid in the presence of magnetic fields. J Magn Magn Mater 324(5):830-842. https://doi. org/10.1016/j.jmmm.2011.09.028

28. Aminfar H, Mohammadpourfard M, Kahnamouei YN (2014) Numerical study of magnetic field effects on the mixed convection of a magnetic nanofluid in a curved tube. Int J Mech Sci 78:81-90. https://doi.org/10.1016/j.ijmecsci.2013.10.014

29. Kalteh M, Hasani H (2014) Lattice Boltzmann simulation of nanofluid free convection heat transfer in an L-shaped enclosure. Superlattices Microstruct 66:112-128. https://doi.org/10.1016/j. spmi.2013.12.004

30. Meyer JP, Adio SA, Sharifpur M, Nwosu PN (2016) The viscosity of nanofluids: a review of the theoretical, empirical, and numerical models. Heat Transfer Eng 37(5):387-421. https:// doi.org/10.1080/01457632.2015.1057447

31. Patel HE, Anoop K, Sundararajan T, Das SK (eds) A micro-convection model for thermal conductivity of nanofluids. In: International heat transfer conference vol 13, Begel House Inc

32. Bejan A (2013) Convection heat transfer. Wiley, Hoboken 
33. Hassani S, Saidur R, Mekhilef S, Hepbasli A (2015) A new correlation for predicting the thermal conductivity of nanofluids; using dimensional analysis. Int J Heat Mass Transf 90:121-130. https:// doi.org/10.1016/j.ijheatmasstransfer.2015.06.040

34. Guo Z, Zheng C, Shi B (2002) Discrete lattice effects on the forcing term in the lattice Boltzmann method. Phys Rev $E$ 65(4):046308. https://doi.org/10.1103/PhysRevE.65.046308

35. Bin D, Bao-Chang S, Guang-Chao W (2005) A new lattice Bhatnagar-Gross-Krook model for the convection-diffusion equation with a source term. Chin Phys Lett 22(2):267. https://doi. org/10.1088/0256-307x/22/2/001

36. White FM, Corfield I (2006) Viscous fluid flow. McGraw-Hill, New York

37. Wang J, Wang M, Li Z (2007) A lattice Boltzmann algorithm for fluid-solid conjugate heat transfer. Int J Therm Sci 46(3):228-234

38. Mohamad AA (2011) Lattice Boltzmann method: fundamentals and engineering applications with computer codes. Springer, Berlin

39. D'Orazio A, Karimipour A, Nezhad AH, Shirani E (2015) Lattice Boltzmann method with heat flux boundary condition applied to mixed convection in inclined lid driven cavity. Meccanica 50(4):945-962. https://doi.org/10.1007/s11012-014-0052-5
40. Lalami AA, Hassanzadeh Afrouzi $H$, Moshfegh A, Omidi M, Javadzadegan A (2019) Investigation of nanofluid heat transfer in a microchannel under magnetic field via lattice Boltzmann method: effects of surface hydrophobicity, viscous dissipation, and joule heating. J Heat Transf 141(6):062403. https://doi. org/10.1115/1.4043163

41. Afshar H, Shams M, Nainian S, Ahmadi G (2009) Microchannel heat transfer and dispersion of nanoparticles in slip flow regime with constant heat flux. Int Commun Heat Mass Transfer 36(10):1060-1066

42. Manay E, Sahin B (2017) Heat transfer and pressure drop of nanofluids in a microchannel heat sink. Heat Transf Eng 38(5):510-522. https://doi.org/10.1080/10407782.2016.11951 62

Publisher's Note Springer Nature remains neutral with regard to jurisdictional claims in published maps and institutional affiliations. 